\title{
BK Virus
}

National Cancer Institute

\section{Source}

National Cancer Institute. BK Virus. NCI Thesaurus. Code C89820.

A double stranded DNA virus in the polyomavirus family. It is widespread but usually causes an asymptomatic infection, except in immunocompromised individuals. 